\title{
Genetic lineage tracing identifies in situ Kit-expressing cardiomyocytes
}

Qiaozhen Liu ${ }^{1, *}$, Rui Yang ${ }^{1, *}$, Xiuzhen Huang ${ }^{1}$, Hui Zhang ${ }^{1}$, Lingjuan $\mathrm{He}^{1}$, Libo Zhang ${ }^{1}$, Xueying Tian ${ }^{1}$, Yu Nie ${ }^{2}$, Shengshou $\mathrm{Hu}^{2}$, Yan Yan ${ }^{3}$, Li Zhang ${ }^{4}$, Zengyong Qiao ${ }^{5}$, Qing-Dong Wang ${ }^{6}$, Kathy O Lui ${ }^{7}$, Bin Zhou ${ }^{1,8,9}$

${ }^{1}$ Key Laboratory of Nutrition and Metabolism, Institute for Nutritional Sciences, Shanghai Institutes for Biological Sciences, Graduate School of Chinese Academy of Sciences, Chinese Academy of Sciences, Shanghai 200031, China, ${ }^{2}$ State Key Laboratory of Cardiovascular Disease, Fuwai Hospital, National Center for Cardiovascular Diseases, Chinese Academy of Medical Sciences and Peking Union Medical College, Beijing 100037, China; ${ }^{3}$ Zhongshan Hospital, Fudan University, Shanghai 200032, China; ${ }^{4}$ Department of Cardiology, The First Affiliated Hospital, School of Medicine, Zhejiang University, 79 Qingchun Road, Hangzhou, Zhejiang 310003, China; ${ }^{5}$ Department of Cardiovascular Medicine, Southern Medical University Affiliated Fengxian Hospital, Shanghai 201499, China; ${ }^{6}$ Cardiovascular and Metabolic Diseases Innovative Medicines, AstraZeneca, Mölndal 43183, Sweden; ${ }^{7}$ Department of Chemical Pathology, Li Ka Shing Institute of Health Sciences, The Chinese University of Hong Kong, Prince of Wales Hospital, Shatin, Hong Kong SAR 999077, China; ${ }^{8}$ Institute of Neuroscience, State Key Laboratory of Neuroscience, CAS Center for Excellence in Brain Science and Intelligence Technology, Shanghai Institutes for Biological Sciences, Chinese Academy of Sciences, Shanghai 200031, China; ' ShanghaiTech University, Shanghai 201210, China

Cardiac cells marked by c-Kit or Kit, dubbed cardiac stem cells (CSCs), are in clinical trials to investigate their ability to stimulate cardiac regeneration and repair. These studies were initially motivated by the purported cardiogenic activity of these cells. Recent lineage tracing studies using Kit promoter to drive expression of the inducible Cre recombinase showed that these CSCs had highly limited cardiogenic activity, inadequate to support efficient cardiac repair. Here we reassess the lineage tracing data by investigating the identity of cells immediately after Cre labeling. Our instant lineage tracing approach identifies Kit-expressing cardiomyocytes, which are labeled immediately after tamoxifen induction. In combination with long-term lineage tracing experiments, these data reveal that the large majority of long-term labeled cardiomyocytes are pre-existing Kit-expressing cardiomyocytes rather than cardiomyocytes formed de novo from CSCs. This study presents a new interpretation for the contribution of Kit ${ }^{+}$cells to cardiomyocytes and shows that Kit genetic lineage tracing over-estimates the cardiogenic activity of Kit ${ }^{+}$CSCs.

Keywords: cardiac stem cell; c-kit; lineage tracing; cardiomyocyte

Cell Research (2016) 26:119-130. doi:10.1038/cr.2015.143; published online 4 December 2015

\section{Introduction}

In the past decade, many preclinical studies have demonstrated the therapeutic potential of stem cells in the treatment of cardiac diseases [1-3]. Some phase 1 clinical trials have reported that infusion of $\mathrm{Kit}^{+}$stem cells in patients with ischemic cardiomyopathy improves

*These two authors contributed equally to this work.

Correspondence: Bin Zhou

Tel: +86-21-54920974

E-mail: zhoubin@sibs.ac.cn

Received 1 September 2015; revised 26 October 2015; accepted 27 October 2015; published online 4 December 2015 left ventricular systolic function and reduces infarct size after myocardial infarction (MI) $[4,5]$, providing a promising hope for future applications of cardiac stem cells (CSCs) in the clinics. In spite of the therapeutic efficacy, the mechanisms of actions, especially the cellular sources for adult heart regeneration, remain controversial [6].

Recently, it has been reported that myocardial injection of lentivirus expressing Cre recombinase driven by Kit promoter into the Rosa26-YFP reporter mice could trace the cell fate of $\mathrm{Kit}^{+}$cells in the injured heart. The authors interpreted that about $8 \%$ cardiomyocytes were derived from resident $\mathrm{Kit}^{+}$cardiac stem cells [7]. The lineage tracing data from the Cre viral injection experi- 
ments were interpreted with an assumption that Kit/Cre is not expressed by cardiomyocytes, so lineage labeling of cardiomyocytes was explained as differentiation from $\mathrm{Kit}^{+}$CSCs. However, it remains debatable whether the regulatory elements used for this Kit promoter are specific for restricting Cre recombinase expression in endogenous Kit-expressing cells [8]. Moreover, the significant contribution of $\mathrm{Kit}^{+}$cells to cardiomyocytes was challenged by a recent non-invasive genetic lineage tracing study using the Kit-MerCreMer and constitutive Kit-Cre mouse lines, which showed that $\mathrm{Kit}^{+}$cells contributed to $<0.03 \%$ cardiomyocytes [9]. In addition, a large portion of labeled cardiomyocytes were derived by fusion of $\mathrm{Kit}^{+}$ cells and pre-existing cardiomyocytes [9], which reduced the percentage of cardiomyocytes differentiated from $\mathrm{Kit}^{+} \mathrm{CSCs}$ to even a smaller number. Such a significant discrepancy in the percentage of Kit-derived cardiomyocytes in the adult heart between these studies [7,9] has sparked debate about the methodologies applied in tracing the cell fate of the $\mathrm{Kit}^{+}$stem cells [10-12].

Here, we independently address this issue using a newly generated Kit-CreER mouse line for inducible lineage tracing. Our instant lineage tracing approach identifies Kit-expressing cardiomyocytes that are labeled during tamoxifen treatment. In combination with longterm lineage tracing experiments, we conclude that the large majority of long-term labeled cardiomyocytes in MI hearts are derived from pre-existing Kit-expressing cardiomyocytes rather than formed de novo from CSCs. This study provides a new interpretation for the lineage tracing results of $\mathrm{Kit}^{+}$cells in the heart, which suggests that Kit genetic lineage tracing over-estimates the cardiogenic activity of $\mathrm{Kit}^{+} \mathrm{CSCs}$.

\section{Results}

$\mathrm{Kit}^{+}$cells contribute minimally to cardiomyocytes during homeostasis

We first crossed Kit-CreER line with Rosa26-RFP reporter line to generate the Kit-CreER;Rosa26-RFP mouse line, and performed tamoxifen administration as previously described [13]. Cre-LoxP recombination-mediated genetic lineage tracing is permanent and irreversible, so any descendants of labeled $\mathrm{Kit}^{+}$cells are tagged with the lineage marker RFP (Figure 1A). Four weeks after tamoxifen treatment, we collected hearts from KitCreER;Rosa26-RFP mice for analysis (Figure 1B). Whole mount fluorescence image showed expression of the lineage marker RFP in some cells of the hearts treated with tamoxifen, and sporadic $\mathrm{RFP}^{+}$cardiomyocytes were found (Figure 1C). In the hearts of control mice that received no tamoxifen, we detected negligible fluo- rescence signals (Figure 1C). To verify the cell identity of these $\mathrm{RFP}^{+}$cells, we performed immunostaining on serial sections of the hearts of Kit-CreER;Rosa26-RFP mice. Immunostaining for the lineage marker RFP, cardiomyocyte marker TNNI3 and endothelial cell marker PECAM on heart sections showed that the Kit-CreERlabeled cells were $\mathrm{TNNI}^{+}$cardiomyocytes or PECAM ${ }^{+}$ endothelial cells (Figure 1D-1F). We have also examined confocal optical sections of the heart and confirmed that these $\mathrm{RFP}^{+}$cells were cardiomyocytes or endothelial cells (Figure $1 \mathrm{G}$ and $1 \mathrm{H}$ ). Immunostaining for an alternative cardiomyocyte marker Desmin and endothelial cell marker VE-cadherin (VE-CAD) in heart sections also showed that $\mathrm{Kit}^{+}$cells contributed to cardiomyocytes and endothelial cells (Supplementary information, Figure S1). We noticed that the majority of $\mathrm{RFP}^{+}$cells adopted an endothelial cell fate in the adult heart during homeostasis $\left(95.13 \% \pm 2.49 \%\right.$ of $\mathrm{RFP}^{+}$cells were RF$\left.\mathrm{P}^{+} \mathrm{PECAM}^{+} ; n=4\right)$. Additionally, we obtained dissociated cardiac cells from Kit-CreER;Rosa26-RFP hearts and detected the individual $\mathrm{RFP}^{+}$cardiomyocyte in vitro (Figure 1I). In the heart sections of Kit-CreER;Rosa26$R F P$ mice that received no tamoxifen, we did not detect any $\mathrm{RFP}^{+}$cardiomyocytes (Supplementary information, Figure S2).

To quantify the percentage of $\mathrm{RFP}^{+}$cardiomyocytes, we performed flow cytometric analysis on the dissociated cardiomyocytes from digested hearts [14]. The dissociated cells were highly enriched for cardiomyocytes (Supplementary information, Figure S3A). We then adopted a cardiomyocyte-specific gating strategy to analyze the percentage of $\mathrm{RFP}^{+}$population among all cardiomyocytes (Supplementary information, Figure S3B and S3C). Our flow cytometric analysis showed that $0.035 \% \pm 0.00088 \%$ cardiomyocytes were $\mathrm{RFP}^{+}$in adult hearts during homeostasis (Figure 1J). In control hearts that received no tamoxifen, $0.0019 \% \pm 0.00016 \%$ cardiomyocytes were $\mathrm{RFP}^{+}$(Figure $1 \mathrm{~J}$ ). This extremely low percentage of $\mathrm{RFP}^{+}$cells in the control hearts was more likely to be the background autofluorescence, as we did not observe a distinct $\mathrm{RFP}^{+}$population and the "RFP" events were distributed next to the gate border (Figure $1 \mathrm{~J})$. In addition, we did not observe any $\mathrm{RFP}^{+}$cardiomyocytes in heart sections from Kit-CreER;Rosa26-RFP mice that received no tamoxifen (Supplementary information, Figure S2). Together, our results demonstrate that $\mathrm{Kit}^{+}$cells contribute minimally to cardiomyocytes in the adult heart during homeostasis.

\section{$\mathrm{Kit}^{+}$cells contribute minimally to cardiomyocytes after injury}

We next tested whether $\mathrm{Kit}^{+}$cells give rise to more 
A

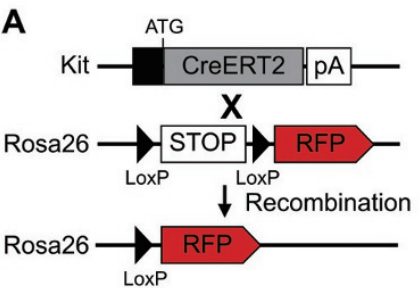

B

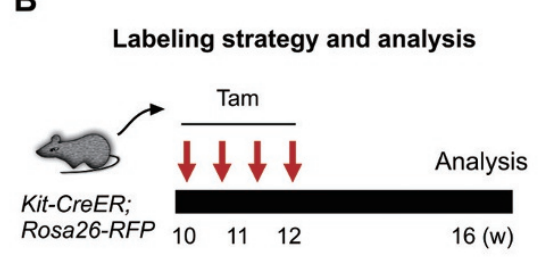

C

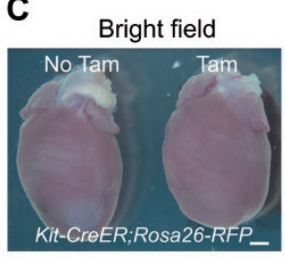

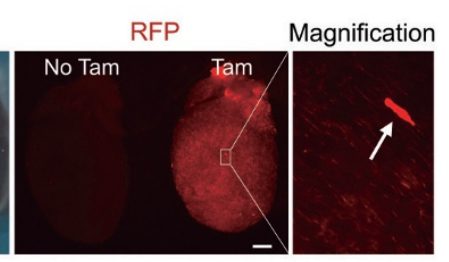
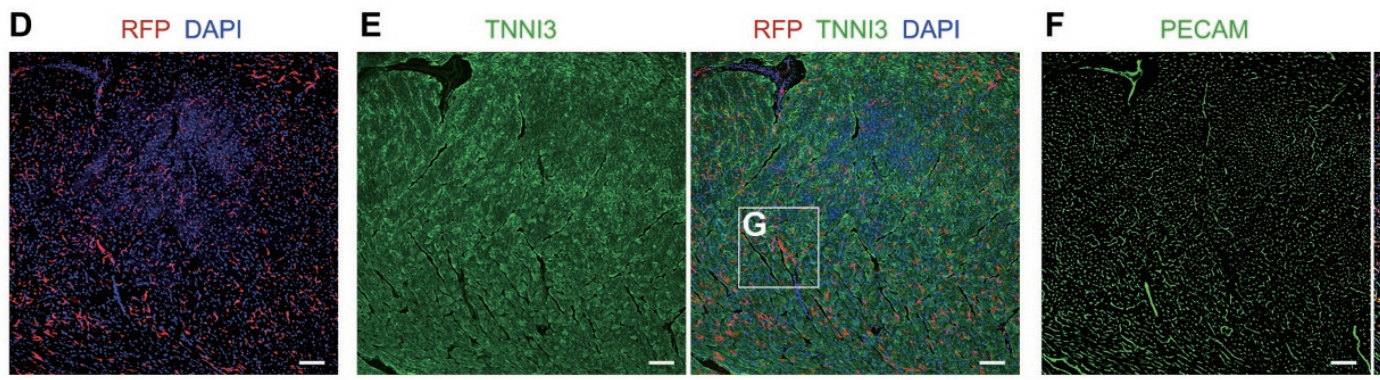

RFP PECAM DAPI
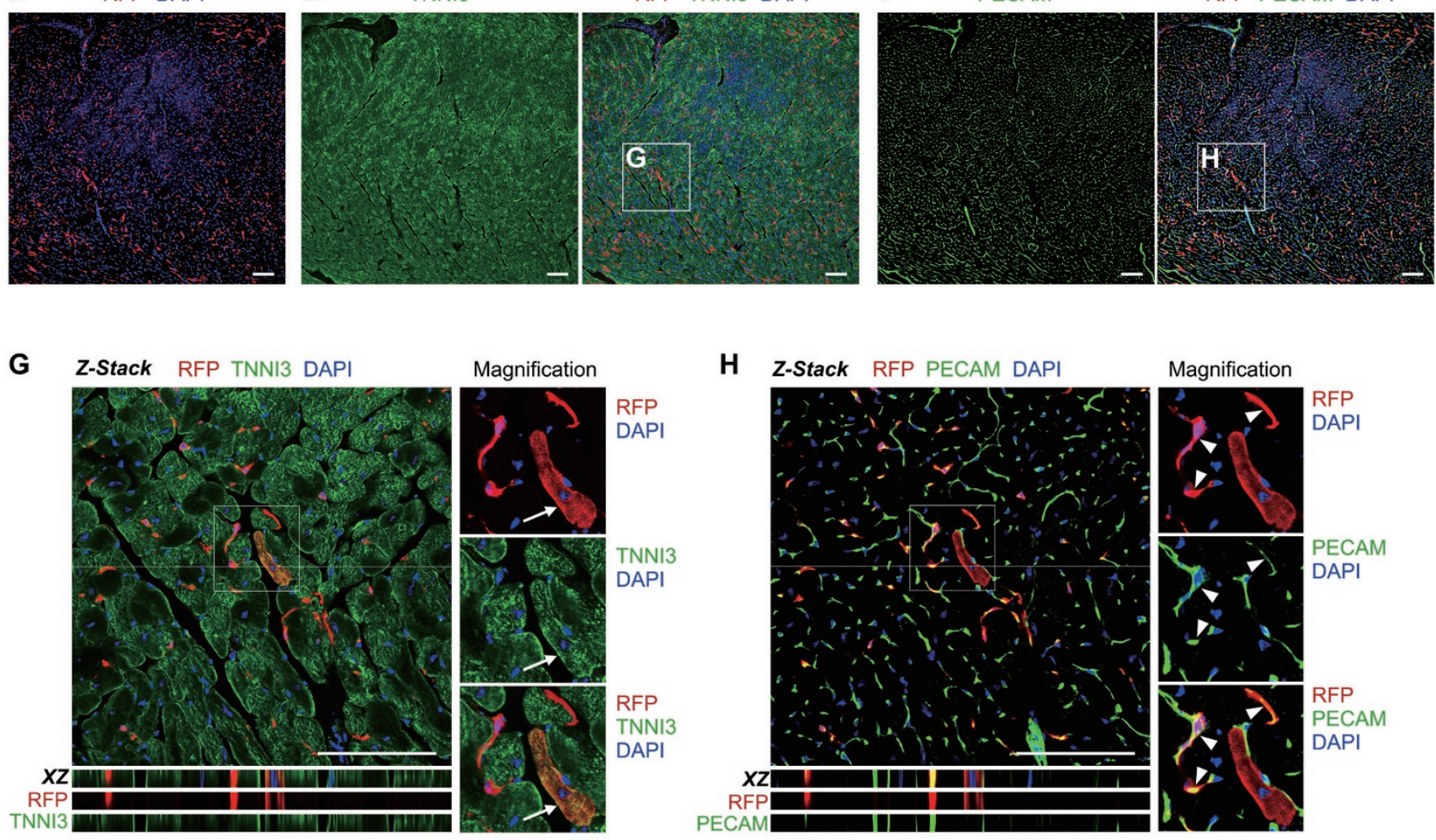

Magnification
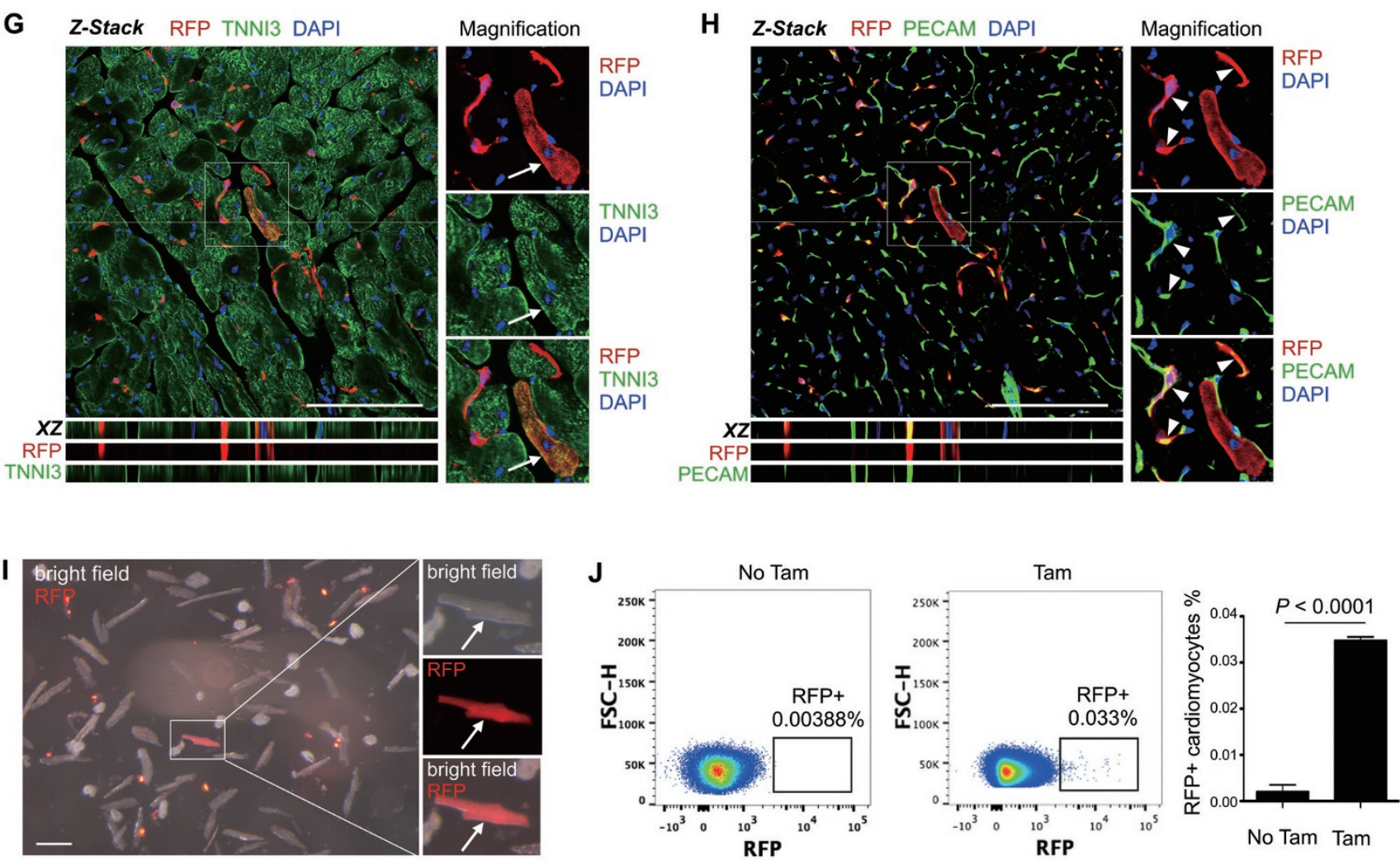

Figure 1 The fate of $\mathrm{Kit}^{+}$cells in heart homeostasis. (A) Kit-CreER mice were crossed with Rosa26-RFP mice for lineage tracing. (B) A schematic of tamoxifen induction and analysis. (C) Whole mount bright field and fluorescence view of KitCreER;Rosa26-RFP hearts with or without tamoxifen (Tam or No Tam). Arrow indicates RFP ${ }^{+}$cardiomyocyte. (D-F) Immunostaining for RFP, TNNI3 and PECAM on Kit-CreER;Rosa26-RFP heart sections. (G, H) Z-stack confocal images including XZ scanned sections showing RFP ${ }^{+} T N N I 3^{+}$cells (arrows, G) and RFP'PECAM ${ }^{+}$cells (arrowheads, $\mathbf{H}$ ). (I) Image of cells dissociated from hearts of Kit-CreER;Rosa26-RFP mice. Arrow indicates a rare $\mathrm{RFP}^{+}$cardiomyocyte. (J) Quantification of $\mathrm{RFP}^{+}$cardiomyocytes by flow cytometry. Events in images are gated on live and lineage-negative cardiomyocytes. $n=4$. Scale bars represent $1 \mathrm{~mm}$ in $\mathbf{C}, 100 \mu \mathrm{m}$ in D-I. 
A

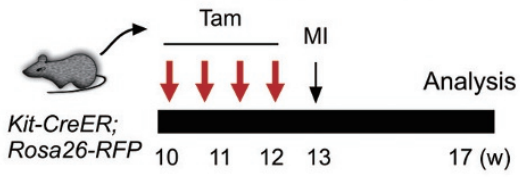

B

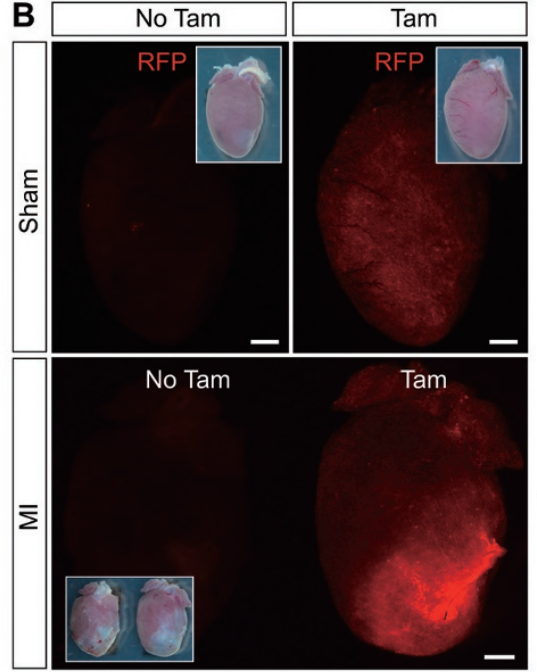

C
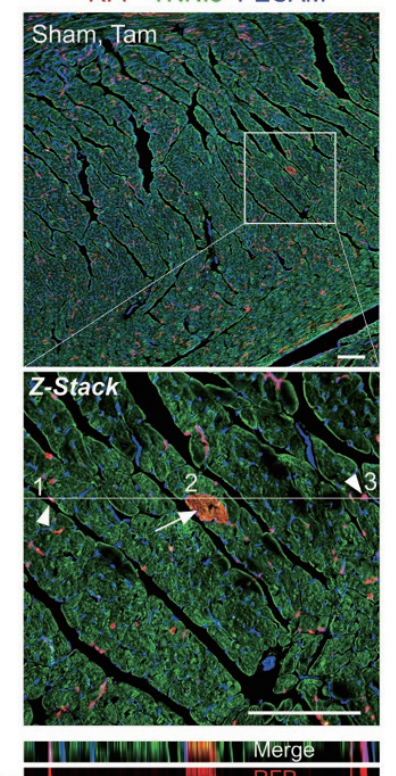

$x Z$

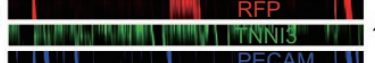

\section{$x$}

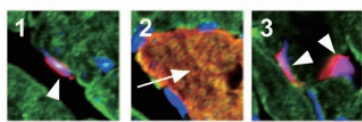

D
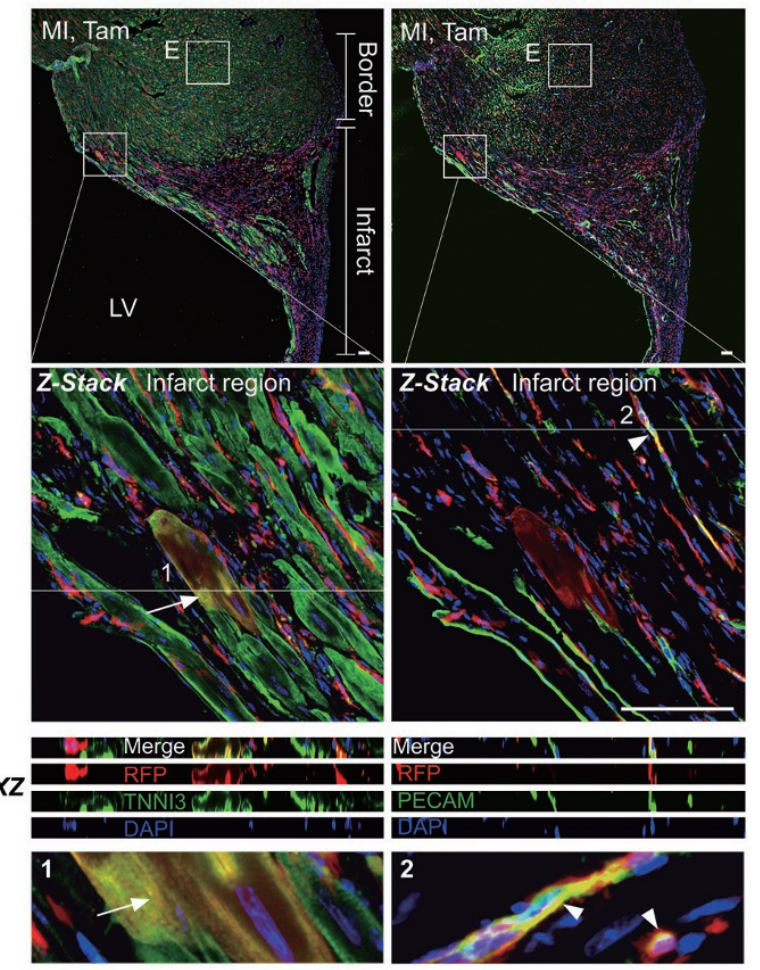

E RFP TNNI3 DAPI
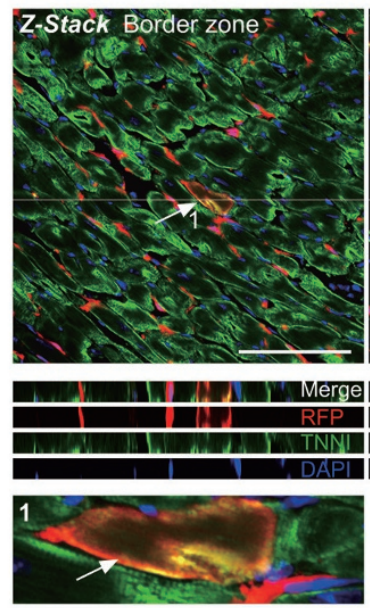

RFP PECAM DAPI

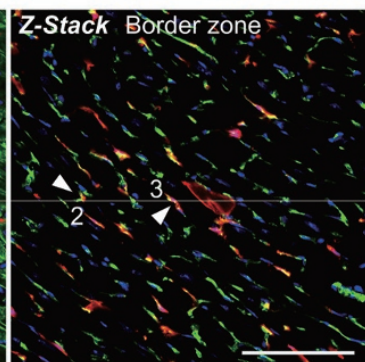

$\mathbf{F}$
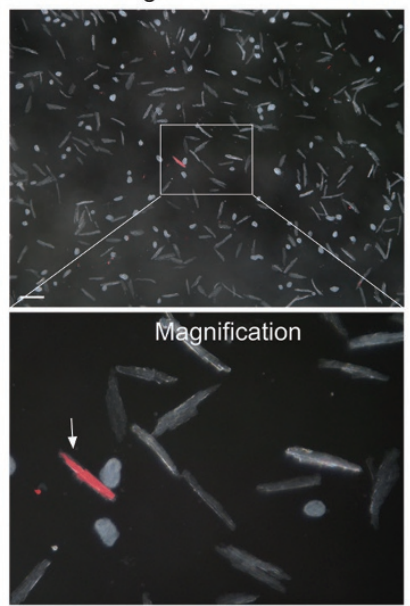

G
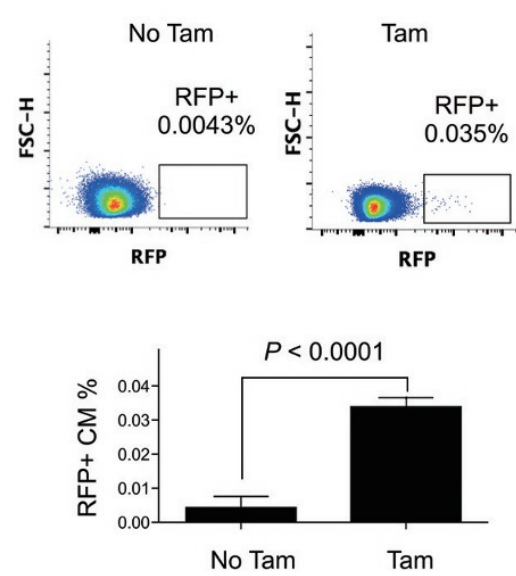

Figure 2 The fate of Kit ${ }^{+}$cells after Ml. (A) A schematic of tamoxifen induction, Ml and analysis. (B) Whole mount fluorescence view of sham or Ml hearts with or without tamoxifen treatment (Tam or No Tam). Inserts are the same hearts in bright field. (C) Immunostaining for RFP, TNNI3 and PECAM on Kit-CreER;Rosa26-RFP heart sections (Sham, Tam). Kit-CreER labels cardiomyocytes (RFP'TNNI3 ${ }^{+}$, arrow) or endothelial cells (RFP'PECAM ${ }^{+}$, arrowheads). (D, E) Immunostaining for RFP, TNNI3 and PECAM on heart sections after MI. Kit-CreER labels cardiomyocytes (RFP ${ }^{+} \mathrm{TNNI}^{+}$, arrow) or endothelial cells $\left(\mathrm{RFP}^{+} \mathrm{PECAM}{ }^{+}\right.$, arrowheads) in both infarcted (D) and border regions (E). Z-stack images were taken for all magnified regions. XZ scanned images are shown by merge and split channels. 1, 2, 3 are magnified images of cells indicated by arrows or arrowheads. (F) Images of cells dissociated from MI hearts of Kit-CreER;Rosa26-RFP mice. Arrow indicates a rare RFP ${ }^{+}$ cardiomyocyte. (G) Quantification of the percentage of RFP ${ }^{+}$cardiomyocytes in MI hearts by flow cytometry. $n=4$. Scale bars, $1 \mathrm{~mm}$ in $\mathbf{B} ; 100 \mu \mathrm{m}$ in $\mathbf{C}-\mathbf{F}$. 
cardiomyocytes after MI. We adopted a similar tamoxifen induction strategy as above, and ligated the left anterior descending (LAD) coronary artery to induce $\mathrm{MI}$ at 1 week after tamoxifen treatment. We analyzed the MI hearts 4 weeks later (Figure 2A). Whole mount fluorescence view of Kit-CreER;Rosa26-RFP hearts showed an increase in the proportion of $\mathrm{RFP}^{+}$cells in the infarcted region, compared to that of the sham-operated hearts (Figure 2B). Immunostaining of RFP, TNNI3 and PECAM on sections of sham-operated and MI hearts of the tamoxifen-treated mice identified $\mathrm{RFP}^{+} \mathrm{TNNI}^{+}$ cardiomyocytes and $\mathrm{RFP}^{+} \mathrm{PECAM}^{+}$endothelial cells in both the sham-operated (Figure 2C) and the infarct and border zone of MI hearts (Figure 2D and 2E). Similar to hearts under homeostasis, we noticed that the majority of $\mathrm{RFP}^{+}$cells adopted an endothelial cell fate in the injured heart, and our quantification showed a significant increase in the number of labeled endothelial cells after injury $(29.13 \pm 2.94$ vs $46.69 \pm 4.62$ cells per field before and after injury, respectively; $n=4$ for each group). By examining the remote zone of MI hearts, we also detected $\mathrm{RFP}^{+} \mathrm{TNNI}^{+}$cardiomyocytes and $\mathrm{RFP}^{+} \mathrm{PECAM}^{+}$ or $\mathrm{RFP}^{+} \mathrm{VE}-\mathrm{CAD}^{+}$endothelial cells (Supplementary information, Figure S4). $0.039 \% \pm 0.011 \%$ of the cardiomyocytes dissociated from the MI hearts were $\mathrm{RFP}^{+}$. Moreover, quantification by flow cytometric analysis of the dissociated cardiomyocytes showed that $0.034 \% \pm$ $0.0015 \%$ cardiomyocytes were $\mathrm{RFP}^{+}$in the MI hearts (Figure 2G). Our results reveal that $\mathrm{Kit}^{+}$cells contribute minimally to cardiomyocytes after heart injury, which is in consistent with the quantification result demonstrated by a recent report [9].

MI does not promote an increase in $\mathrm{Kit}^{+}$cardiomyocytes after injury

To compare the percentage of $\mathrm{Kit}^{+}$cells contributing to cardiomyocytes before and after LAD coronary artery ligation, we quantified the percentage of labeled cardiomyocytes by flow cytometry (Figure $3 \mathrm{~A}$ ). We did not find a higher percentage of $\mathrm{RFP}^{+}$cardiomyocytes in the MI hearts compared with that of the sham-operated hearts (Figure 3B), suggesting that MI does not promote an increased number of Kit-derived cardiomyocytes. Nevertheless, MI increased the number of $\mathrm{RFP}^{+} \mathrm{PECAM}^{+}$ endothelial cells as shown above. In addition, we noticed more $\mathrm{RFP}^{+} \mathrm{CD} 11 \mathrm{~b}^{+}$macrophages and $\mathrm{RFP}^{+} \mathrm{FSP}^{+}$fibroblasts in the infarcted regions of injured heart, compared with that of the sham-operated hearts (Supplementary information, Figure S5). There was also no significant increase in the percentage of $\mathrm{RFP}^{+}$cardiomyocytes 5 weeks after tamoxifen induction in both the sham-operated and MI hearts compared with that at 1 week after tamoxifen induction (Figure 3B), suggesting no significant increase in the differentiation of $\mathrm{Kit}^{+}$cells into cardiomyocytes during the 4-week tracing period. This surprising result prompted us to investigate the identity of Kit-CreERlabeled cells before injury. To achieve this, we performed short-term lineage tracing experiments by collecting KitCreER;Rosa26-RFP hearts 1 week after tamoxifen induction (before operation group). We could readily detect $\mathrm{RFP}^{+}$cardiomyocytes by whole mount fluorescence microscopy (Figure 3C). Immunostaining for RFP, TNNI3 and PECAM on heart sections showed the presence of $\mathrm{RFP}^{+} \mathrm{TNNI}^{+}$cardiomyocytes in addition to $\mathrm{RFP}^{+} \mathrm{PE}-$ $\mathrm{CAM}^{+}$endothelial cells (Figure 3D). Examination of the dissociated cardiac cells confirmed the identity of $\mathrm{RFP}^{+}$ cardiomyocytes (Figure 3E). Collectively, our results suggest that most $\mathrm{RFP}^{+}$cardiomyocytes, if not all, were genetically labeled within the first week after tamoxifen treatment. In another words, there would be a negligible amount of $\mathrm{Kit}^{+}$cells differentiating into cardiomyocytes in the next 4 weeks after sham or MI operation.

\section{Instant lineage tracing strategy identifies Kit-expressing cardiomyocytes}

Our short-term lineage tracing experiments in the before operation group suggest a possibility that most RFP cardiomyocytes in the lineage tracing experiments are likely pre-existing cardiomyocytes rather than derived from $\mathrm{Kit}^{+} \mathrm{CSC}$ s through lineage conversion. To test this hypothesis, we performed instant-chase experiments, in which hearts were collected within the first $48 \mathrm{~h}$ after tamoxifen treatment. We used bone marrow (BM) cells to test the labeling efficiency and specificity by instant labeling (Supplementary information, Figure S6). We found that $>50 \% \mathrm{Kit}^{+}$cells were $\mathrm{RFP}^{+}$(efficiency) and $>90 \% \mathrm{RFP}^{+}$cells were $\mathrm{Kit}^{+}$(specificity). With this instant-chase strategy, we observed $\mathrm{RFP}^{+} \mathrm{TNNI}^{+}$cardiomyocytes in Kit-CreER;Rosa26-RFP hearts (Figure 4A). From tamoxifen gavage to RFP signal detection, a series of biological processes are required, which include at least four steps: tamoxifen absorption, CreER translocation into the nucleus, Cre-mediated DNA recombination, RFP transcription and translation to certain level that allows detection by fluorescence microscopy. The remaining time for the presumed labeled $\mathrm{Kit}^{+} \mathrm{CSCs}$ to differentiate into cardiomyocytes, if any, is very limited in this scenario. Therefore, the detection of $\mathrm{RFP}^{+}$cardiomyocytes within such a short time window is unlikely due to the differentiation of $\mathrm{Kit}^{+} \mathrm{CSCs}$ into cardiomyocytes through lineage conversion. To further examine whether Kit-CreER labels Kit-expressing cardiomyocytes during lineage tracing, we collected Kit-CreER;Rosa26-RFP hearts within the first $24 \mathrm{~h}$ after tamoxifen treatment. 
A
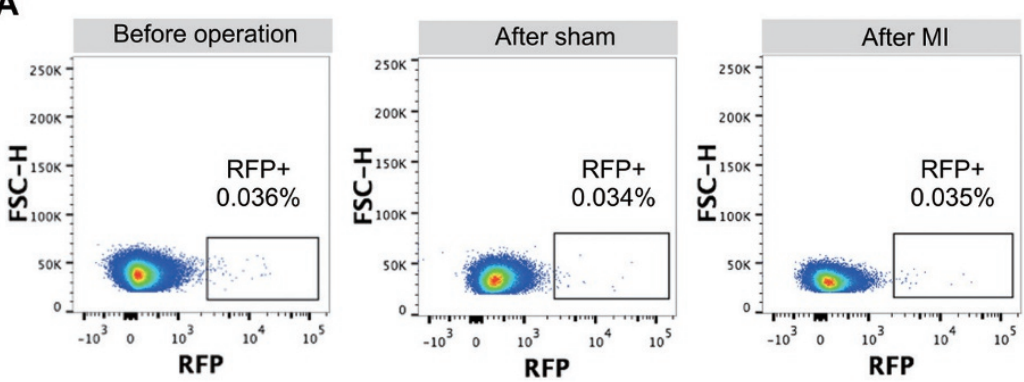

B

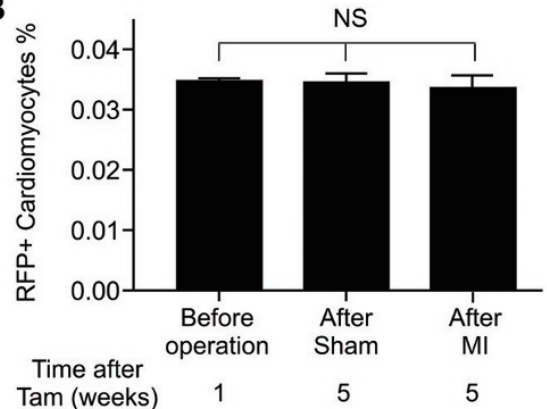

C

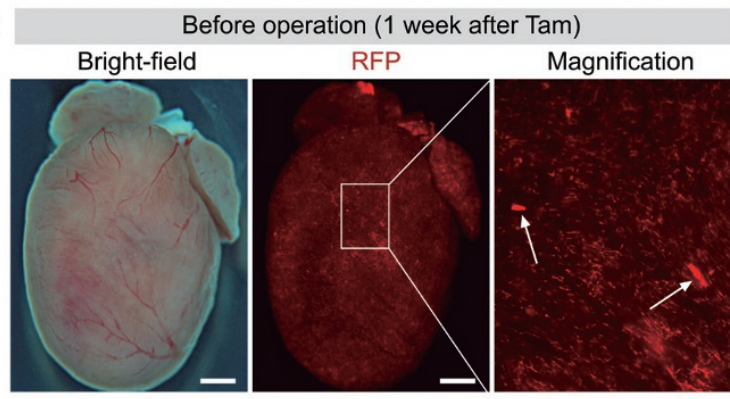

E

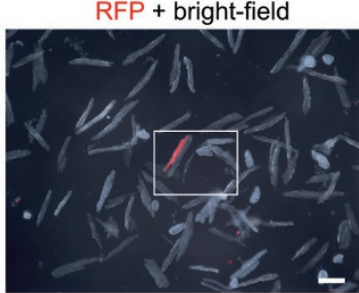

Magnification

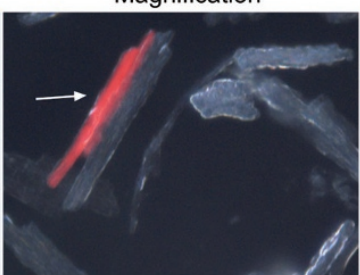

D
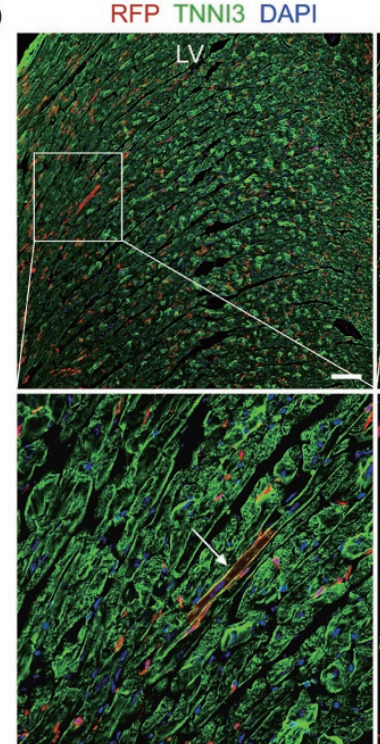

RFP PECAM DAPI
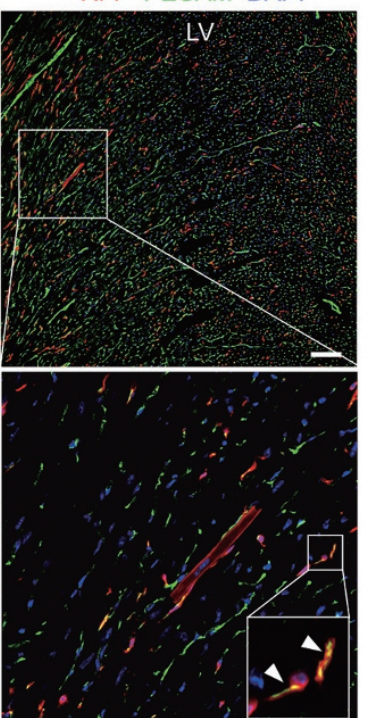

Figure 3 Quantification of Kit-CreER-labeled cardiomyocytes before and after MI. (A) Flow cytometric analysis of RFP ${ }^{+}$cardiomyocytes isolated from Kit-CreER;Rosa26-RFP mouse hearts. (B) Quantification of the percentage of RFP ${ }^{+}$cardiomyocytes labeled by Kit-CreER. $n=4$; NS, non-significant. (C) Whole mount bright field and fluorescence view of Kit-CreER;Rosa26-RFP mouse heart. Arrows indicate RFP ${ }^{+}$cardiomyocytes. (D) Immunostaining for RFP, TNNI3 and PECAM in heart sections reveals $\mathrm{RFP}^{+}$cardiomyocyte (arrow) and endothelial cells (arrowheads). LV, left ventricle. (E) Image of cells dissociated from Kit-CreER;Rosa26-RFP mouse hearts ( $n=3$ hearts analyzed). Arrow indicates a RFP ${ }^{+}$cardiomyocyte. Scale bars, 1 $\mathrm{mm}$ in $\mathbf{C}, 100 \mu \mathrm{m}$ in $\mathbf{D}$ and $\mathbf{E}$.

With this instant-chase strategy, we repeatedly observed $\mathrm{RFP}^{+} \mathrm{TNNI}^{+}$cardiomyocytes in mouse hearts (Figure 4B). To avoid artifacts due to weaker RFP signals in instant tracing study compared to the long-term lineage tracing experiments, we examined the dissociated cardiac cells and found $\mathrm{RFP}^{+}$cardiomyocytes among them (Figure 4C). Quantification of the number of Kit-CreERlabeled cardiomyocytes by immunostaining showed that instant-chase strategy (24-48 h) identified $58 \%$ of labeled cardiomyocytes in the MI hearts (Figure 4D). Quantification of the number of Kit-CreER-labeled cardiomyocytes by flow cytometric analysis showed that instant-chase strategy (48 h) identified $71 \%$ of the labeled cardiomyo- cytes in the MI hearts (Figure 4E). If $\mathrm{Kit}^{+} \mathrm{CSC}$ differentiate into new cardiomyocytes after tamoxifen treatment (e.g., 5 weeks later), it is unlikely that $58 \%-71 \%$ of the cardiomyocytes are differentiated from the $\mathrm{Kit}^{+} \mathrm{CSCs}$ within the first $48 \mathrm{~h}$. Our instant lineage tracing experiments, therefore, identify a pre-existing population of Kit-expressing cardiomyocytes in the adult heart, which may account for the large majority of labeled cardiomyocytes during lineage tracing.

\section{Existence of Kit-expressing cardiomyocytes in adult hearts}

To directly visualize the Kit-expressing cardiomy- 
A

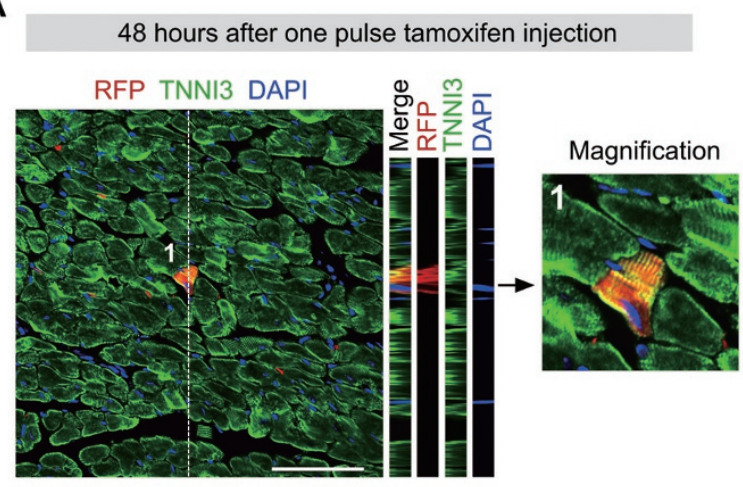

B

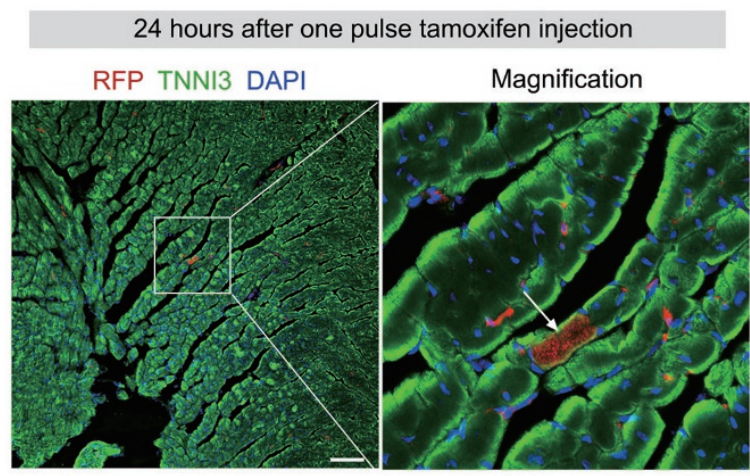

C
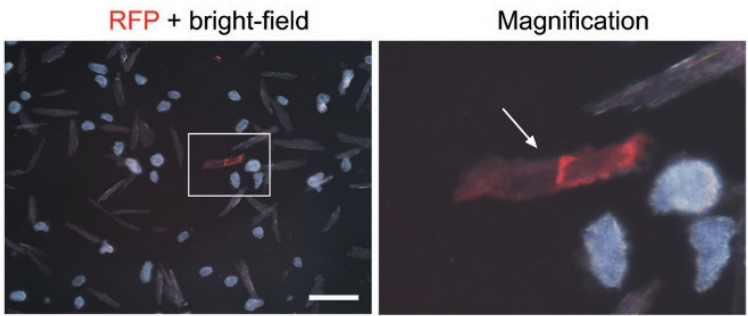
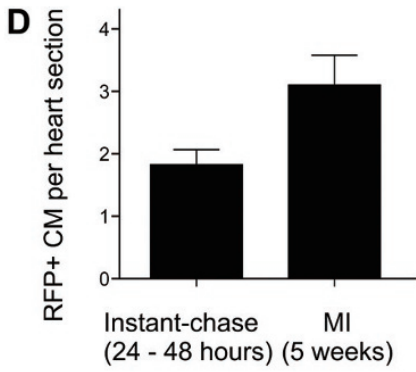

E

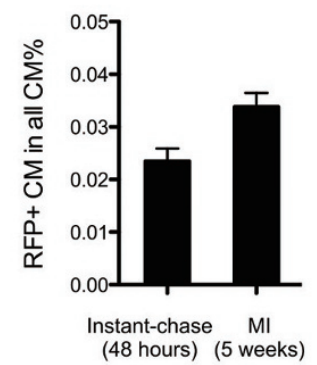

Figure 4 Instant lineage tracing reveals Kit-expressing cardiomyocytes. (A) Z-stack confocal images showing RFP and TNNI3 in heart sections from Kit-CreER;Rosa26-RFP mice at $48 \mathrm{~h}$ after tamoxifen injection. (B) Immunostaining for RFP and TNNI3 in heart sections from mice collected $24 \mathrm{~h}$ after tamoxifen injection. (C) Images of heart cells isolated from mice at $24 \mathrm{~h}$ after tamoxifen injection. Arrow indicates the RFP ${ }^{+}$cardiomyocyte. (D) Quantification of RFP ${ }^{+}$cardiomyocytes (CM) per heart sections in instant lineage tracing heart samples (24-48 h after tamoxifen treatment) versus hearts after MI (5 weeks after tamoxifen treatment). For each heart, 80 to 100 slides were examined for quantification, and 4-6 hearts from each group were analyzed. (E) Quantification of the percentage of RFP ${ }^{+}$cardiomyocytes by flow cytometry. $n=4$. Scale bar, $100 \mu \mathrm{m}$.

ocytes, we utilized the Kit-BAC-GFP transgenic mice where GFP is used as a surrogate for Kit expression [15]. We could observe $\mathrm{GFP}^{+}$cardiomyocytes from the whole mount fluorescence view (Figure 5A). By dissociation of cardiac cells, we also detected $\mathrm{GFP}^{+}$cardiomyocytes among isolated cardiomyocytes in vitro (Figure 5B). Immunostaining for GFP and TNNI3 on heart sections of Kit-BAC-GFP mice showed $\mathrm{GFP}^{+} \mathrm{TNNI}^{+}$cardiomyocytes, confirming the existence of Kit-expressing cardiomyocytes in the adult heart (Figure 5C and 5D), which is in line with the observations in the neonatal mouse hearts reported previously by other groups $[15,16]$.

To independently address whether Kit is expressed by adult cardiomyocytes, we performed immunostaining with KIT antibody on cardiomyocytes isolated from wild-type mice. We found a few $\mathrm{TNNI}^{+}$cardiomyocytes expressing weak but detectable Kit protein (Figure 6A and $6 \mathrm{~B})$. To further validate whether the instant-chased $\mathrm{RFP}^{+}$cardiomyocytes expressed Kit, we performed immunostaining for RFP and Kit with primary cardio- myocytes dissociated from the Kit-CreER;Rosa26-RFP mice that received one dose of tamoxifen. We found a few $\mathrm{RFP}^{+}$cardiomyocytes expressing Kit (Figure 6C), indicating that the Kit-CreER-labeled cardiomyocytes is likely due to the in situ Kit expression by adult cardiomyocytes.

\section{Discussion}

Recently, there is a heated debate over the proportion of cardiomyocytes differentiated from $\mathrm{Kit}^{+} \mathrm{CSCs}$ after injury $[7,9,10,12]$. The lineage tracing data based on our Kit-CreER model show that $\mathrm{Kit}^{+}$cells contribute to as few as $\sim 0.035 \%$ cardiomyocytes of the entire heart during cardiac homeostasis and after injury, which is consistent with a recent report by Molkentin's group [9]. In our study, we do not find any increase in differentiation of $\mathrm{Kit}^{+}$cells to cardiomyocytes after injury. Intriguingly, we observe an increase in the population of Kit-derived endothelial cells and inflammatory cells in the injured 
A

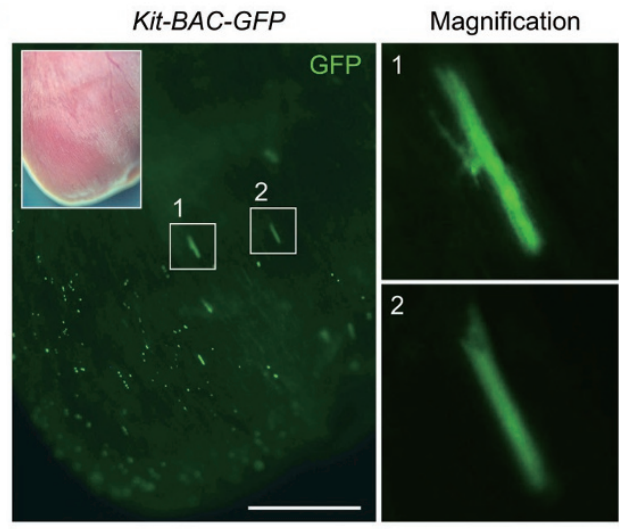

B

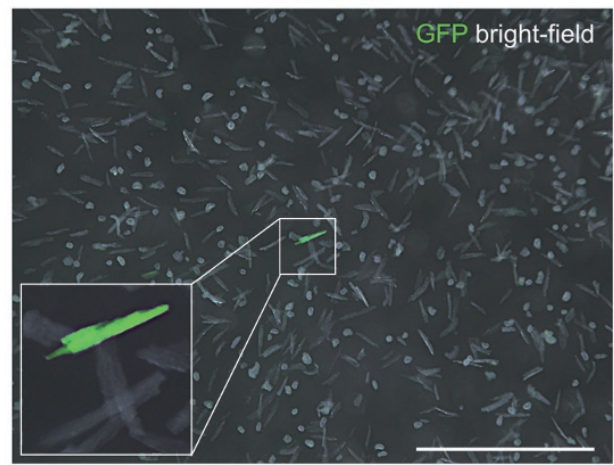

C
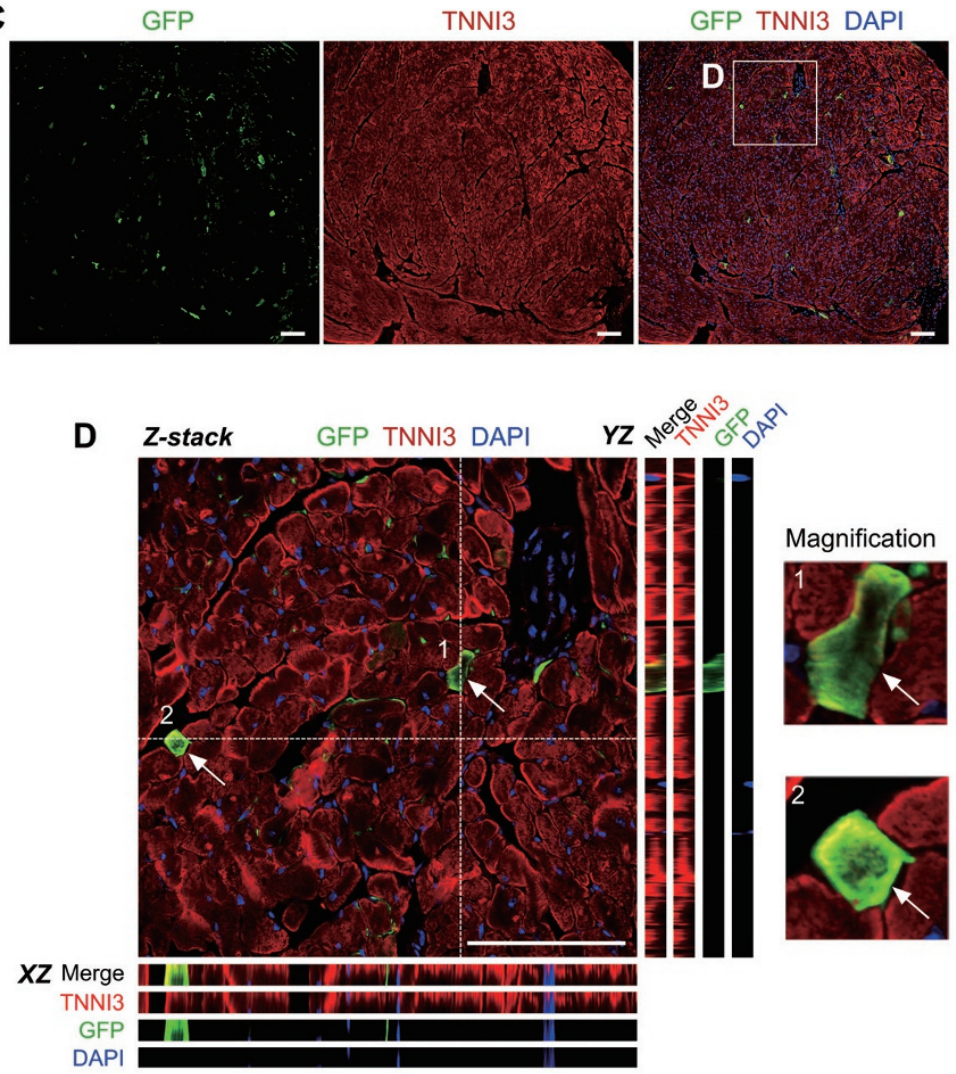

Figure $\mathbf{5}$ Kit is in situ expressed by cardiomyocytes in the adult heart. (A) Whole mount fluorescence view of the adult Kit$B A C$-GFP heart. Boxed regions are magnified in right panels. GFP is a surrogate for Kit. (B) Image of isolated cells from the adult Kit-BAC-GFP heart shows $\mathrm{Kit}^{+}$cardiomyocytes. (C) Immunostaining for GFP and TNNI3 on heart sections of Kit-BACGFP mice. (D) Z-stack confocal images along the $X Z$ and $Y Z$ axes. 1 and 2 denote two Kit ${ }^{+}$cardiomyocytes (arrows). Images are representatives of three individual samples. Scale bars, $1 \mathrm{~mm}$ in $\mathbf{A}$ and $\mathbf{B}, 100 \mu \mathrm{m}$ in $\mathbf{C}$ and $\mathbf{D}$.

heart. Our results in the short-term vs long-term lineage tracing experiments suggest that the majority of labeled cardiomyocytes are restricted at the stage shortly after tamoxifen treatment (short-term) rather than the result of differentiation of $\mathrm{Kit}^{+} \mathrm{CSCs}$ afterwards (long-term). The instant tracing experiments identified Kit-expressing cardiomyocytes in the hearts, which surprisingly represented about $50 \%-70 \%$ of those labeled cardiomyocytes in the MI hearts. Our work presents a new explanation for Kit-derived cardiomyocytes in in-depth genetic lineage tracing studies.

Interpretation of results from lineage tracing studies largely relies on localization of Cre and the specificity of promoter-driven expression of Cre. The positive lineage tracing data in a previous report were interpreted as differentiation of non-cardiomyocyte $\mathrm{Kit}^{+} \mathrm{CSCs}$ into cardiomyocytes [7]. This was largely based on the assumption that Kit is not expressed by the pre-existing cardiomyocytes of the adult hearts. However, due to the lack of a good antibody for Cre detection in the heart, and the possible weak expression of Kit in cardiomyocytes, it is difficult to reach an unambiguous conclusion for Cre expression. The weak Cre expression, although difficult to detect, may catalyze Cre-LoxP recombination that leads to the binary readout of the reporter, e.g. RFP. Therefore, the argument of "negative" Kit expression in cardiomyocytes requires more careful examinations and any positive results from lineage tracing studies as such, require more cautious interpretation. Since the readout of fate mapping is binary and easy to detect, we take advantage of the Kit-CreER model for instant "pulseand-chase" experiments to label possible Kit-expressing cardiomyocytes during the first $24 \mathrm{~h}$. These labeled cells likely represent the pre-existing Kit-expressing cardiomyocytes, although confirmation by immunostaining for Cre is technically difficult to achieve. Most importantly, the number of $\mathrm{RFP}^{+}$cardiomyocytes labeled by the instant-chasing approach represents more than half of the $\mathrm{RFP}^{+}$cardiomyoytes in the MI hearts. Taking into account that the long-term lineage tracing experiments 
A

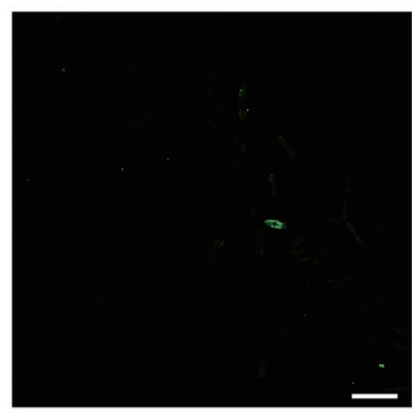

B

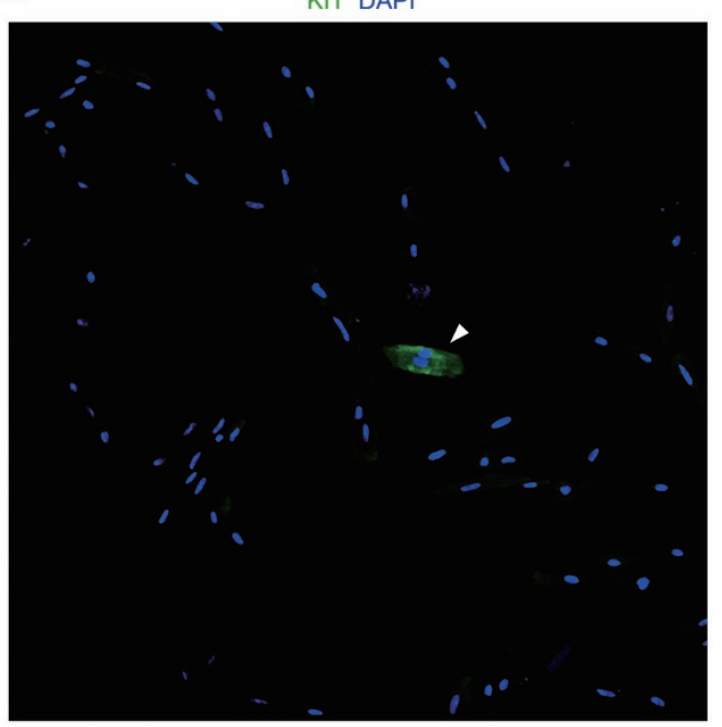

DAPI

KIT TNNNI3 DAPI
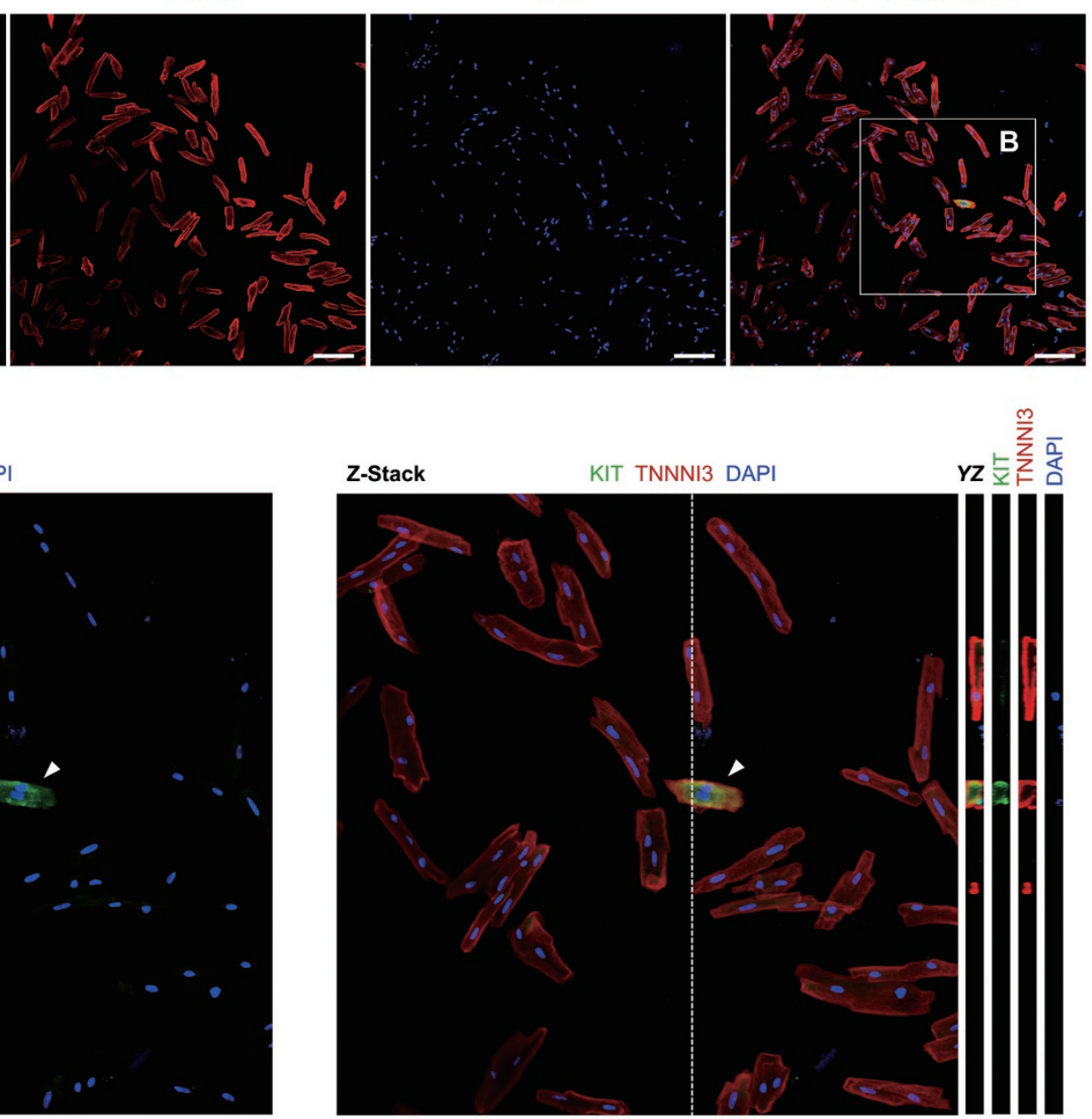

C

C RFP
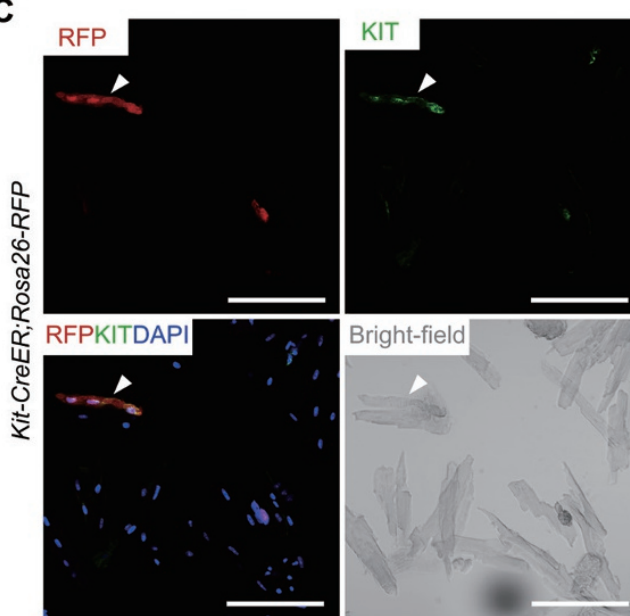

DAPI
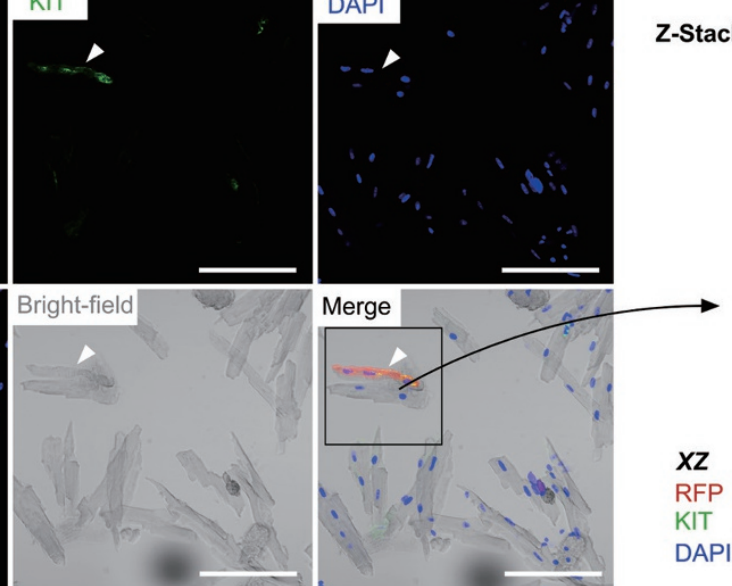

$X Z$

RFP

KIT

DAPI

Figure 6 Kit is expressed by cardiomyocytes. (A) Immunostaining for Kit and TNNI3 on cardiomyocytes isolated from wild type heart. (B) Averaged intensity from projection of 9 optical sections; YZ sections (from dotted lines) are also shown. Arrowhead indicates $\mathrm{Kit}^{+}$cardiomyocyte. (C) Immunostaining for RFP and Kit on cardiomyocytes isolated from Kit-CreER;Rosa26RFP mice treated with tamoxifen. Averaged intensity from projection of 9 optical sections; $X Z$ and $Y Z$ sections (from dotted lines) are also shown. Scale bar, $100 \mu \mathrm{m}$. 
involve multiple injections of tamoxifen over the course of 5 weeks, the number of Kit-expressing cardiomyocytes in the short-term instant tracing (24-48 h) with a single-pulse tamoxifen injection could be underestimated. Therefore, it is likely that most, if not all, Kit-CreER labeled cardiomyocytes in the MI hearts are derived from pre-existing cardiomyocytes marked immediately after tamoxifen treatment.

Indeed, it has been reported that Kit is transiently expressed by cardiovascular progenitors during embryonic heart development. A subset of $\mathrm{Nkx} 2-5^{+}$cardiovascular progenitors also express Kit, and these $\mathrm{Nkx} 2-5^{+} \mathrm{Kit}^{+}$cardiovascular progenitors possess the capacity of long-term self-renewal and differentiation into both cardiomyocytes and smooth muscle cells in vitro [17]. In another study, the $\mathrm{Nkx} 2-5^{+} \mathrm{Kit}^{+}$cells were reported to represent a multipotent precursor population that is capable of differentiating into the major cardiac lineages including endothelial cells, in addition to cardiomyocytes and smooth muscle cells [18]. Similar to the embryonic stage, Kit expression identifies cardiovasular progenitors in the neonatal heart that could differentiate into cardiomyocytes and endothelial cells [15]. These $\mathrm{Kit}^{+}$progenitors support myogenesis in the neonatal heart following myocardial infarction; however, such a regenerative capacity is lost in the adult heart [19]. Compared with the neonatal stage, Kit-expressing cardiomyocytes are rarely found in the adult heart [16]. Therefore, consistent with previous work, our instant lineage tracing data show that only a small subset of cardiomyocytes $(\sim 0.03 \%)$ express Kit in the adult heart. The reduction of Kit-expressing cardiomyocytes is coincident with the onset of terminal differentiation that happens from the neonatal to adult stage, indicating the possibility that expression of Kit could promote cardiac stem cell differentiation and regulate terminal differentiation [20]. Whether expression of Kit in a subset of cardiomyocytes promotes de-differentiation and subsequent re-differentiation in vivo nevertheless warrants further investigation, as a recent in vitro study has demonstrated that Kit expression is significantly upregulated during the de-differentiation process [21].

In summary, our work does not support the interpretation that most of the labeled cardiomyocytes in lineage tracing are formed de novo by lineage conversion of $\mathrm{Kit}^{+} \mathrm{CSCs}$ [7]. Instead, we suggest that the majority of labeled cardiomyocytes are derived from pre-existing Kit-expressing cardiomyocytes immediately after tamoxifen induction.

\section{Materials and Methods}

\section{Animals}

All animals were used according to the guidelines of Institutional Animal Care and Use Committee of the Institute for $\mathrm{Nu}$ tritional Sciences, Shanghai Institutes for Biological Sciences, Chinese Academy of Sciences. Kit-CreER, Rosa26-RFP and Kit$B A C-G F P$ mice were reported previously $[13,15,22]$. Tamoxifen (Sigma, T5648) was dissolved in corn oil $(20 \mathrm{mg} / \mathrm{ml})$ and administered by gavage at the indicated time points $(0.1-0.15 \mathrm{mg}$ tamoxifen/g mouse body weight) as described previously [13]. Both male and female mice were used in our experiments and randomized into different treatment groups. The researchers were not blinded to mice of the tamoxifen untreated group, before operation group, sham and myocardial infarction groups.

\section{Myocardial infarction}

Myocardial infarction was performed as described previously [23]. Briefly, mice were anesthetized with isoflurane in an airtight chamber, and their chest hair was shaved afterwards. The chest was then disinfected with iodine and mice were placed on a heated pad in supine position. The mice were ventilated and anesthetized with trachea cannula, which was connected to a respiratory machine (Harvard Apparatus) and an anesthetic gas machine (Harvard Apparatus). The breathing of mice was maintained at about 120 140 breaths per min. An incision of $\sim 1 \mathrm{~cm}$ was made between the third and fourth ribs and left thoracotomy was made to expose the mouse heart. Left anterior descending (LAD) coronary artery was then ligated with a suture at the upper $1 / 3$ location. The chest and skin were closed, and disinfected with iodine again. The respiratory machine was kept on to ventilate mice with oxygen until the mice woke up. We collected mouse hearts 3 to 7 weeks after myocardial infarction for analyses of the fate of $\mathrm{Kit}^{+}$cells. In the sham operation group, same procedure was followed except ligation of the LAD coronary artery was omitted. All operation procedures were performed according to animal protocols.

\section{Whole mount fluorescence microscopy}

Collected mouse hearts were washed in PBS and placed on agar for the whole mount bright field and fluorescence imaging using the Zeiss (AxioZoom V16) or Leica stereo microscope (Leica M165 FC). To determine magnification of specific regions, we used the automated Z-stack images acquired by the Zeiss stereoscope (AxioZoom V16).

\section{Immunostaining}

Immunostaining was performed according to protocols described previously [24]. Briefly, mouse hearts were collected at the indicated time points and fixed in $4 \%$ PFA for about $1 \mathrm{~h}$ at $4{ }^{\circ} \mathrm{C}$. After three washes in PBS, the hearts were dehydrated in $30 \%$ sucrose overnight until they sank to the bottom. Following a $1 \mathrm{~h}$ immersion in optimum cutting temperature (O.C.T., Sakura) at $4{ }^{\circ} \mathrm{C}$, the hearts were embedded in blocks and frozen at $-80^{\circ} \mathrm{C}$. Sections of $10 \mu \mathrm{m}$ thickness were collected on slides and blocked in $5 \%$ PBSST (5\% normal donkey serum in PBS with $0.1 \%$ triton X-100) for $30 \mathrm{~min}$ at room temperature. The slides were then incubated with primary antibodies at $4{ }^{\circ} \mathrm{C}$ overnight and protected from light. The primary antibodies and dilution are: TNNI3 (Abcam, ab56357, 1:200), RFP (Rockland, 600401379, 1:1 000), PECAM (BD Pharmingen, 553370, 1:500), Desmin (R\&D, AF3844, 1:100) VE-cadherin (R\&D, AF1002, 1:200), CD11b (BD Pharmingen, 550282, 1:100), FSP1 (Dako, A5114, 1:100), GFP (Invitrogen, 
A11122, 1:200). In the next day, slides were washed with PBS for three times and then incubated with secondary antibodies for about $30 \mathrm{~min}$ at room temperature in dark. After three washes in PBS, we used Alexa Fluor fluorescent antibodies (Invitrogen) for detection of the primary antibody. For weak signals, horseradish peroxidase-conjugated antibodies (Jackson ImmunoResearch) and tyramide signal amplification kit (PerkinElmer) were used to expose the signals as previously described [25]. All slides were mounted with fluorescence-protecting mounting medium containing nuclei dye DAPI (Vector Lab). Images were taken by Olympus confocal microscopy system (FV1200) or Zeiss confocal microscopy system (LSM710). To provide Z-stack confocal images, we scanned 5-9 consecutive XY images on the $z$ axis with confocal microscopy. The obtained images were analyzed by ImageJ (NIH) software. The images were merged using the Image color-merge channels function, and the stacks were performed using Z-projects and average intensity projection. In the stack, orthogonal view was performed to reveal the signals on the XZ and YZ axes. Merged signals and split channels are shown to delineate the signals at single cell resolution, as described previously [13].

\section{Cardiomyocyte quantification}

The whole heart was sectioned and mounted on 50 to 60 slides, with each containing 8-10 heart tissue sections. About 80100 heart tissue sections representatively spanning each heart were stained with TNNI3, RFP and PECAM. The number of TN$\mathrm{NI}^{+}{ }^{\mathrm{RFP}^{+}}$cells was quantified under the Olympus fluorescence microscope (BX53). The total number of $\mathrm{TNNI}^{+} \mathrm{RFP}^{+}$cells from 80 to 100 heart tissue sections was divided by the total number of heart sections examined, obtaining the number of $\mathrm{TNNI}^{+} \mathrm{RFP}^{+}$ cardiomyocytes per heart tissue section. For different groups, 4-6 hearts in each group were quantified for comparison.

\section{Dissociated adult cardiomyocyte preparation}

Adult cardiomyocytes were isolated as described previously [14]. Briefly, we injected Kit-CreER;Rosa26-RFP mice with 200 $\mu 1$ heparin $(6.25 \mathrm{U} / \mu \mathrm{l})$ intraperitoneally. Thirty minutes later, $10 \%$ chloral hydrate was injected intraperitoneally to anaesthetize mice. Dissected hearts were retrograde perfused with modified Tyrode's solution (MTS) for 30 to $60 \mathrm{~min}$. The hearts were next perfused with MTS containing $250 \mathrm{U} / \mathrm{ml}$ collagenase type 2 (Worthington, LS004176) and 0.3 U/ml Protease XIV (Sigma, P5147) to dissociate heart tissues. Digested heart tissues were minced by forceps and filtered through $100 \mu \mathrm{m}$ strainer. Isolated cells were centrifuged at $20 \times \mathrm{g}$ for $2 \mathrm{~min}$ at $4{ }^{\circ} \mathrm{C}$ to allow most of the cardiomyocytes to be collected. The supernatant was collected and cell pellets that contained cardiomyocytes were re-suspended in MTS with $1 \%$ BSA for further analysis. Non-cardiomyocytes from supernatant were obtained by centrifuge at $500 \times g$ for 3 min at $4{ }^{\circ} \mathrm{C}$.

\section{Isolated cardiomyocyte staining}

Cardiomyocytes were isolated from C57/B6 mice or KitCreER;Rosa26-RFP mice as described above. After obtaining the cell pellet, the collected cardiomyocytes were incubated with increasing concentrations of calcium ion in four steps. In details, cells were re-suspended with $0.5 \mathrm{mg} / \mathrm{ml} \mathrm{BSA}$ in $\mathrm{CaCl}_{2}(0.06 \mathrm{mM}$, $0.24 \mathrm{mM}, 0.6 \mathrm{mM}$ and $1.2 \mathrm{mM}$ )-containing MTS. Each step took $4 \mathrm{~min}$. Cells were next re-suspended in $1.2 \mathrm{mM}$ calcium chloride/10\% FBS-containing alpha-MEM (Invitrogen) and plated on laminin (Invitrogen)-coated coverslips (Fisher or NEST) in 24-well cell culture plate (Corning) for at least $4 \mathrm{~h}$. After well attached to the coverslips, cells were fixed in 4\% PFA for $15 \mathrm{~min}$ and then blocked in blocking buffer (5\% normal donkey serum in PBS with $0.5 \%$ triton $\mathrm{X}-100$ ) for $30 \mathrm{~min}$ at room temperature. Subsequently, cells were incubated with primary antibodies (c-kit, R\&D, AF1356, 1:50; TNNI3, Santa Cruz, sc-15368, 1:100; RFP, Rockland, 600401379, 1:1 000) at $4{ }^{\circ} \mathrm{C}$ for $24 \mathrm{~h}$ and then gently washed in PBS. Alexa Fluor fluorescent secondary antibodies (Invitrogen) were used to detect the signals. Following wash out of the secondary antibodies, the coverslips were mounted with fluorescence-protecting mounting medium (Vector Lab) and images were taken on an Olympus confocal microscopy system (FV1200).

\section{Bone marrow (BM) cells isolation and flow cytometry}

Mouse hind limbs were collected and bisected by cutting through the knee joint. Muscle and connective tissues of both the femur and tibia were removed by scraping the diaphysis of the bone. After cleaning, bones were rinsed in ice-cold 70\% ethanol for 10-15 min and washed in cold PBS for $5 \mathrm{~min}$ for three times. The femur and tibia were cut just at the end of the marrow cavity and the marrow was sluiced out by RPMI 1640 (Invitrogen) containing $10 \%$ FBS and $2 \mathrm{mM}$ EDTA followed by filtering through $100 \mu \mathrm{m}$ filter. Cells were centrifuged at $2000 \times \mathrm{g}$ for $10 \mathrm{~min}$ and red blood cells were lysed with RBC lysis buffer (Roche). Washed and collected cells from previous step were purified by $40 \% / 80 \%$ percoll, and stained sequentially with Violet Dye and antibody cocktail for flow cytometry analysis. The antibody cocktail for different lineage markers included CD3e-PECy7 (eBioscience, 25-0031), CD19-PECy7 (eBioscience, 25-0193), CD11b-PECy7 (eBioscience, 25-0112), Ly6G-PECy7 (eBioscience, 25-5931), Ter119-PECy7 (eBioscience, 25-5921). The cells were stained spontaneously with an antibody against Kit/CD117-APC (eBioscience, 17-1171, 1:200).

\section{Flow cytometry}

Dissociated cardiomyocytes were first stained with the LIVE/ DEAD Fixable Violet Dead Cell Stain Kit (Life Technology, L34955) according to the manufacturer's instruction. $500 \mu \mathrm{l}$ isolation buffer ( 2 mM EDTA and $0.5 \%$ BSA in PBS) was added to wash the cells, followed by centrifugation for $3 \mathrm{~min}$ at $20 \times \mathrm{g}$. These cardiomyocytes were subsequently stained with antibody mixture (Lin) containing CD3e PE-Cy7 (eBioscience, 25-0031), CD19 PE-Cy7 (eBioscience, 25-0193), CD11b PE-Cy7 (eBioscience, 25-0112), CD11c PE-Cy7 (eBioscience, 25-0114), Ly-6G (Gr-1) PE-Cy7 (eBioscience, 25-5931), Ter-119 PE-Cy7 (eBioscience, 25-5921) at a dilution of 1:200 for $30 \mathrm{~min}$ at $4{ }^{\circ} \mathrm{C}$. Finally, the cells were re-suspended in $500 \mu 1$ isolation buffer. The stained cells were analyzed using a FACS Aria II Flow Cytometer (BD Bioscience), and the raw data processed by Flowjo (TreeStar) according to protocols described previously [13].

\section{Statistical analysis}

All data were obtained from four to six independent experiments as indicated in each figure legend and are presented as mean values \pm SEM. All mice were randomly assigned to different experimental groups. Statistical comparisons between datasets were made with analysis of normality and variance. A two-sided unpaired Student's $t$-test was used for comparing differences between 
two groups, and ANOVA test was used for comparing differences between three or more groups. The null hypothesis was rejected if $P$ was $<0.05$.

\section{Acknowledgments}

We thank Prof Hongkui Zeng for sharing the Rosa26-RFP mouse line and Shanghai Biomodel Organism Co., Ltd. for generating Kit-CreER mouse line. This work was supported by Shanghai Zhangjiang Stem Cell Research Project (ZJ2014-ZD-002), Ministry of Science and Technology (2013CB945302, 2012CB945102), the National Natural Science Foundation of China (91339104, 91339102, 31271552, 31222038, 31301188, 81430006, 31571503, 31501172), Youth Innovation Promotion Association of CAS (2015218), Shanghai Basic Research Key Project (14JC1407400), Shanghai Institutes for Biological Sciences (SIBS) President Fund, Sanofi-SIBS Fellowship, AstraZeneca, Shanghai Yangfan Project (15YF1414000), Qimingxing Project (15QA1404300) and Research Grants Council of Hong Kong (24110515).

\section{References}

1 Anversa P, Kajstura J, Rota M, Leri A. Regenerating new heart with stem cells. J Clin Invest 2013; 123:62-70.

2 Garbern JC, Lee RT. Cardiac stem cell therapy and the promise of heart regeneration. Cell Stem Cell 2013; 12:689-698.

3 Loffredo FS, Steinhauser ML, Gannon J, Lee RT. Bone marrow-derived cell therapy stimulates endogenous cardiomyocyte progenitors and promotes cardiac repair. Cell Stem Cell 2011; 8:389-398.

4 Chugh AR, Beache GM, Loughran JH, et al. Administration of cardiac stem cells in patients with ischemic cardiomyopathy: the SCIPIO trial: surgical aspects and interim analysis of myocardial function and viability by magnetic resonance. Circulation 2012; 126:S54-S64.

5 Bolli R, Chugh AR, D'Amario D, et al. Cardiac stem cells in patients with ischaemic cardiomyopathy (SCIPIO): initial results of a randomised phase 1 trial. Lancet 2011; 378:18471857.

6 van Berlo JH, Molkentin JD. An emerging consensus on cardiac regeneration. Nat Med 2014; 20:1386-1393.

7 Ellison GM, Vicinanza C, Smith AJ, et al. Adult c-kit(pos) cardiac stem cells are necessary and sufficient for functional cardiac regeneration and repair. Cell 2013; 154:827-842.

8 Molkentin JD, Houser SR. Are resident c-kit+ cardiac stem cells really all that are needed to mend a broken heart? Circ Res 2013; 113:1037-1039.

9 van Berlo JH, Kanisicak O, Maillet M, et al. c-kit+ cells minimally contribute cardiomyocytes to the heart. Nature 2014; 509:337-341.

10 Nadal-Ginard B, Ellison GM, Torella D. Absence of evidence is not evidence of absence: pitfalls of cre knock-ins in the c-Kit locus. Circ Res 2014; 115:415-418.

11 Molkentin JD. Letter by Molkentin regarding article, "The absence of evidence is not evidence of absence: the pitfalls of Cre Knock-Ins in the c-Kit Locus". Circ Res 2014; 115:e21-e23.

12 Torella D, Ellison GM, Nadal-Ginard B. Adult c-kitpos Cardiac Stem Cells Fulfill Koch's Postulates as Causal Agents for Cardiac Regeneration. Circ Res 2014; 114:e24-e26.

13 Liu Q, Huang X, Zhang H, et al. c-kit(+) cells adopt vascular endothelial but not epithelial cell fates during lung maintenance and repair. Nat Med 2015; 21:866-868.

14 Nakayama H, Bodi I, Correll RN, et al. alphalG-dependent T-type $\mathrm{Ca} 2+$ current antagonizes cardiac hypertrophy through a NOS3-dependent mechanism in mice. J Clin Invest 2009; 119:3787-3796.

15 Tallini YN, Greene KS, Craven M, et al. c-kit expression identifies cardiovascular precursors in the neonatal heart. Proc Natl Acad Sci USA 2009; 106:1808-1813.

16 Zaruba MM, Soonpaa M, Reuter S, Field LJ. Cardiomyogenic potential of C-kit(+)-expressing cells derived from neonatal and adult mouse hearts. Circulation 2010; 121:1992-2000.

17 Wu SM, Fujiwara Y, Cibulsky SM, et al. Developmental origin of a bipotential myocardial and smooth muscle cell precursor in the mammalian heart. Cell 2006; 127:1137-1150.

18 Christoforou N, Miller RA, Hill CM, Jie CC, McCallion AS, Gearhart JD. Mouse ES cell-derived cardiac precursor cells are multipotent and facilitate identification of novel cardiac genes. J Clin Invest 2008; 118:894-903.

19 Jesty SA, Steffey MA, Lee FK, et al. c-kit+ precursors support postinfarction myogenesis in the neonatal, but not adult, heart. Proc Natl Acad Sci USA 2012; 109:13380-13385;

20 Li M, Naqvi N, Yahiro E, et al. c-kit is required for cardiomyocyte terminal differentiation. Circ Res 2008; 102:677685.

21 Kubin T, Poling J, Kostin S, et al. Oncostatin $\mathrm{m}$ is a major mediator of cardiomyocyte dedifferentiation and remodeling. Cell Stem Cell 2011; 9:420-432.

22 Madisen L, Zwingman TA, Sunkin SM, et al. A robust and high-throughput Cre reporting and characterization system for the whole mouse brain. Nat Neurosci 2010; 13:133-140.

23 Zhou B, Honor LB, He H, et al. Adult mouse epicardium modulates myocardial injury by secreting paracrine factors. $J$ Clin Invest 2011; 121:1894-1904.

24 Tian X, Hu T, Zhang H, et al. Subepicardial endothelial cells invade the embryonic ventricle wall to form coronary arteries. Cell Res 2013; 23:1075-1090.

25 Liu Q, Hu T, He L, et al. Genetic targeting of sprouting angiogenesis using Apln-CreER. Nat Commun 2015; 6:6020.

(Supplementary information is linked to the online version of the paper on the Cell Research website.)

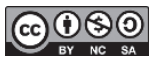

This license allows readers to copy, distribute and transmit the Contribution as long as it attributed back to the author. Readers are permitted to alter, transform or build upon the Contribution as long as the resulting work is then distributed under this is a similar license. Readers are not permitted to use the Contribution for commercial purposes. Please read the full license for further details at - http://creativecommons.org/ licenses/by-nc-sa/4.0/ 